\title{
Association Between Anthropometric Measurements and Dietary Habits in a Young Adult Female Population in Selected Areas in the Southern Province of Sri Lanka
}

\author{
Samamalee Upekshi Kankanamge ${ }^{1,}$, Sameera Ariyarathna ${ }^{2}$, Poruthotage Pradeep Rasika Perera ${ }^{3}$ \\ ${ }^{1}$ Department of Pharmacy, Faculty of Allied Health Sciences, General Sir John Kotelawala Defence University, Werahera, Sri Lanka \\ ${ }^{2}$ Department of Allied Health Sciences, Faculty of Medical Sciences, University of Sri Jayewardenepura, Gangodawila, Sri Lanka \\ ${ }^{3}$ Department of Biochemistry, Faculty of Medical Sciences, University of Sri Jayewardenepura, Gangodawila, Sri Lanka
}

Email address:

samamalee@ymail.com (S. U. Kankanamge), sameera.ariyarathna@gmail.com (S. Ariyarathna), rasika@sjp.ac.lk (P. P. R. Perera)

${ }^{*}$ Corresponding author

\section{To cite this article:}

Samamalee Upekshi Kankanamge, Sameera Ariyarathna, Poruthotage Pradeep Rasika Perera. Association Between Anthropometric Measurements and Dietary Habits in a Young Adult Female Population in Selected Areas in the Southern Province of Sri Lanka. International Journal of Science and Qualitative Analysis. Vol. 2, No. 3, 2016, pp. 24-29. doi: 10.11648/j.ijsqa.20160203.11

Received: October 8, 2016; Accepted: November 2, 2016; Published: November 30, 2016

\begin{abstract}
Obesity and abdominal obesity are becoming major public health problem in certain countries. Causes for obesity are multi-factorial; among them various dietary factors play an important role. The main cause of obesity-related deaths is cardiovascular disease, for which abdominal obesity is a predisposing factor [1]. Abdominal obesity is defined as Waist to Hip Ratio (WHR) above 0.90 for males and above 0.85 for females [2]. Body Mass Index (BMI) is a parameter that is useful in assessing obesity, overweight and malnutrition. The study aimed to assess the association between anthropometric measurements and the dietary habits in a female population of two study areas. A cross-sectional study was conducted separately at Hungama and Hiththatiya-Middle areas within Hambantota and Matara districts of Southern province of Sri Lanka. 304 healthy females between 20-50 years of age were selected by systematic random sampling method. For the calculation of BMI and WHR, height and weight, Waist Circumference (WC) and Hip Circumference (HC) of each individual were measured. The World Health Organization cut-off points were used to categorize BMI and WHR. An interviewer administered questionnaire was used to assess dietary habits. There is a statistically significant difference between the WHR and consumption of potato $(p=0.002)$ in Hungama as well as significant difference can be seen between WHR and consumption of red raw rice $(p=0.022)$ and white raw rice $(p=0.020)$ in Hiththatiya middle. Present study shows statistically positive relationship between the BMI and consumption of potato $(p=0.002)$ yogurt $(p=0.046)$ and fried food $(p=0.033)$ in the total study population. Prevalence of obesity (6.6\%) and abdominal obesity $(47 \%)$ in whole population are higher with compared with other similar studies [3].
\end{abstract}

Keywords: Obesity, Abdominal Obesity, Dietary Habits, Female

\section{Introduction}

This study was conducted to investigate the prevalence of obesity, abdominal obesity using anthropometric parameters and their variations with dietary patterns in two rapidly developing administrative units in Southern Province of Sri Lanka. Obesity and abdominal obesity are becoming major public health concerns in South Asia, and even the prevalence rates are higher in affluent countries [4]. These
Asian countries also face a grave burden of obesity-related disorders such as diabetes, hypertension, and cardiovascular diseases. Changes in dietary habits and physical activity have been implicated as potential causes of obesity. With changing food habits and increasingly sedentary lifestyles, the prevalence of obesity has increased markedly in certain Asian countries over recent decades [5]. Obesity among female population has been increasing at an alarming rate throughout the world in the recent past. Abdominal obesity or increased visceral adipose tissue is a more common condition among 
the female population. Both generalized and abdominal obesity are associated with increased risk of morbidity and mortality. The main cause of obesity-related deaths is cardio vascular disease, for which abdominal obesity is a predisposing factor [1]. Because of that, abdominal adiposity reflected by waist-hip ratio may be an important parameter in assessing health risk of Asian population. WHR (Waist to Hip Ratio) is the waist circumference divided by the hip circumference and it has been suggested as an additional measure of body fat distribution. It looks at the proportion of fat stored on the body around the waist and hip [6]. Abdominal obesity is defined as Waist to Hip Ratio (WHR) above 0.90 for males and above 0.85 for females [2]. Recent studies have shown that waist circumference (WC) is the best simple anthropometric index of abdominal visceral adipose tissue, and may also be the best index for predicting cardiovascular risks [7]. Few studies have compared the degree of association of WHR and WC with cardiovascular risks after adjustment for total fat or BMI (Body Mass Index). BMI is a parameter that is useful in assessing obesity and malnutrition. It is calculated as "weight in kilograms divided by height in meters squared $\left(\mathrm{kg} / \mathrm{m}^{2}\right)$ " [1]. According to the WHO, cut-off points for BMI are, $18 \cdot 5-24.9 \mathrm{~kg} / \mathrm{m}^{2}$ (normal range), $>25 \mathrm{~kg} / \mathrm{m}^{2}$ (overweight), $>30 \mathrm{~kg} / \mathrm{m}^{2}$ (obesity).

Sri Lanka is a low-middle income country with undergoing rapid epidemiological and nutritional transition. Compared with males, females are significantly more sedentary, much less physically active and have fewer intakes of foods. Therefore, women are more affected with obesity and nutritional related disease risks. The economic transition that many developing countries are undergoing is producing important changes in diet and lifestyle that greatly impact on obesity and obesity related disease risks. Despite considerable research effort, the nutritional etiology of obesity remains unclear and controversial, especially with regard to the roles of dietary fat and carbohydrate [8]. Thus the study was conducted to assess the prevalence of obesity and abdominal obesity as a baseline to observe its variations, if any, in the two selected administrative units within the Hambantota and Matara districts in Sri Lanka, in the future.

\section{Methods}

\subsection{Subject}

The study was conducted in Hungama and Hiththatiya middle in two administrative units in the Hambantota and Matara districts of the Southern Province of Sri Lanka. Healthy females in the age group 20-50 years were randomly selected to the study as the study population. All healthy females in the age group of 20-50 years who gave consent were taken into the study and pregnant women, females who have chronic diseases (e.g. Diabetes Mellitus, Hypertension) and females who were suffering from acute infections (e.g. fever) were excluded. The Obesity, overweight and abdominal obesity status was assessed using anthropometric parameters such as BMI and WHR.

\subsection{Sampling}

The houses were selected by systematic random sampling. Every third house in the grama niladhari list was selected. Subsequently, the selection of the individuals for the study was done by visiting those selected homes. From each household, one to three healthy individuals in the age group of 20-50 years were selected randomly. The number of healthy females selected from each grama niladhari area was 152. A sample of 304 healthy females representing both Hiththatiya middle and Hungama areas were recruited to this study. The prevalence of abdominal obesity in adult Sri Lankan population was reported as $26.2 \%$ in recent sudy conducted in Sri Lanka [3]. Therefore, Sample size was targeted for a $26.2 \%$ prevalence rate within a $95 \%$ confidence interval and it was estimated at 152 . Therefore, from each grama niladhari area, 152 individuals were randomly selected to achieve a sample of 304 females.

\subsection{Data Collection}

Interviewer administered questionnaires were used to assess dietary habits of the study population. Informed consent was obtained from all individuals selected for the study before interviewing and taking body measurements. Body measurements were taken at their home in a private place without disclosing to outsiders with minimal contact. For the calculation of BMI, height (m) and weight $(\mathrm{kg})$ and for the calculation of WHR, waist circumference (WC) and hip (HC) circumference of each individual were measured. Waist Circumference was measured at the approximate midpoint between the lower margin of the least palpable rib and the top of the iliac crest, using a stretch-resistant tape and Hip circumference was measured around the widest portion of the buttocks, with the tape parallel to the floor following WHO criteria.

\subsection{Ethical Approval}

The study protocol was approved by the Ethical Review Committee of the Faculty of Medical sciences, University of Sri Jayewardenepura, Sri Lanka. The participants were fully explained about the nature of the study and informed written consent were obtained. The privacy of the research participants and the confidentiality of data provided by them were completely protected. Data was securely stored and was available only to the investigator/supervisor and data is not divulged to any third party. Participants were entirely free to clarify any problems they had regarding the study at any time. Individuals found as obese, centrally obese and malnourished were educated on healthy lifestyle diets and encouraged to decrease the consumption of starchy foods and fried foods to decrease the risk of obesity and consequent health risks.

\subsection{Statistical Analysis}

Data analysis was done using a computer based statistical 
package, SPSS (version 15). Descriptive statistical methods were used to describe and summarize the sample characteristics. Pearson's chi-square test were used as significant tests as when required. A p-value of $<0.05$ was taken as the significant probability level.

\section{Results}

The age of the study population ranged from 20-50 years. Average age of the subjects was 38 years. The total number of individuals who participated in the study was 304 . From each administrative area 152 individuals participated.

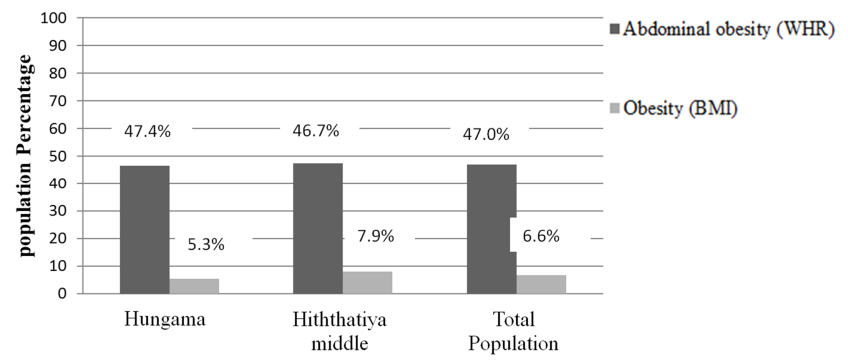

Figure 1. Distribution of obesity and abdominal obesity in two grama niladhari areas.

Abdominal obesity is mainly defined by the WC and obesity is defined by the BMI. Risk of diseases or fat distribution is defined by the WHR. Persons with abdominal obesity as well as obesity as defined by WC and BMI were comparatively less in Hiththatiya Middle than Hungama. The population with risk of diseases is comparatively higher in Hungama (47.4\%) than Hiththatiya Middle (46.7\%). This shows considerably high prevalence in obese $(6.6 \%)$ and abdominal obesity $(47.0 \%)$ in the total population considering BMI and WHR.

Table 1. Anthropometric characteristics of participants in two areas.

\begin{tabular}{llll}
\hline Variable & Hiththatiya Middle & Hungama & p-value* \\
\hline BMI & & & \\
Mean & 23.21 & 23.78 & 0.666 \\
SD & 3.90 & 4.07 & \\
WHR & & & \\
Mean & 0.85 & 0.85 & \\
SD & 6.52 & 5.85 & 0.909 \\
\hline
\end{tabular}

*Comparing Hiththatiya and Hungama areas (based on Pearson chi-square test)

Mean BMI and WHR values of both areas are in the normal cutoff range. BMI is $18 \cdot 5-24 \cdot 9 \mathrm{~kg} / \mathrm{m}^{2}$ is considered as normal. Waist to Hip Ratio (WHR) above 0.85 for females is considered as abnormal.

Table 2. Comparing BMI with dietary habits in the total population.

\begin{tabular}{|c|c|c|c|c|c|c|c|c|c|c|c|c|c|}
\hline \multirow{2}{*}{ Food } & \multicolumn{3}{|c|}{ Underweight } & \multicolumn{3}{|c|}{ Normal } & \multicolumn{3}{|c|}{ Overweight } & \multicolumn{3}{|c|}{ Obesity } & \multirow{2}{*}{ P-value } \\
\hline & $\mathbf{0}$ & 1 & 2 & $\mathbf{0}$ & 1 & 2 & $\mathbf{0}$ & 1 & 2 & $\mathbf{0}$ & 1 & 2 & \\
\hline RED RAW RICE & 2 & 4 & 22 & 3 & 37 & 135 & 0 & 10 & 71 & 0 & 4 & 16 & 0.115 \\
\hline WHITE RAW RICE & 4 & 18 & 6 & 14 & 123 & 38 & 10 & 61 & 10 & 0 & 16 & 4 & 0.319 \\
\hline FISH & 2 & 5 & 21 & 4 & 27 & 144 & 3 & 15 & 63 & 1 & 0 & 19 & 0.375 \\
\hline RED MEAT & 28 & 0 & 0 & 172 & 3 & 0 & 77 & 4 & 0 & 18 & 2 & 0 & 0.096 \\
\hline WHITE MEAT & 5 & 22 & 1 & 18 & 154 & 3 & 14 & 67 & 0 & 3 & 17 & 0 & 0.464 \\
\hline POTATO & 0 & 6 & 10 & 0 & 50 & 56 & 0 & 15 & 125 & 0 & 26 & 16 & 0.002 \\
\hline LEAVES & 0 & 5 & 23 & 0 & 20 & 155 & 0 & 6 & 75 & 0 & 4 & 16 & 0.277 \\
\hline FRUIT & 0 & 1 & 27 & 0 & 3 & 172 & 0 & 6 & 75 & 0 & 1 & 19 & 0.153 \\
\hline YOGHURT & 14 & 12 & 0 & 60 & 42 & 2 & 34 & 108 & 5 & 8 & 17 & 2 & 0.046 \\
\hline FRIED FOOD & 20 & 11 & 13 & 10 & 13 & 30 & 23 & 70 & 30 & 19 & 40 & 25 & 0.033 \\
\hline VEGETABLE OIL & 28 & 0 & 0 & 171 & 3 & 1 & 79 & 2 & 0 & 19 & 1 & 0 & 0.879 \\
\hline
\end{tabular}

(Consumption frequency: $0=$ Never $1=3$ days or less than 3 days/week $2=$ more than 3 days/week)

Regarding dietary habits in the total population, there is a statistically significant difference between consumption of certain food types and BMI. Consumption of red raw rice, white raw rice, red meat, grain, fruits and vegetable oil does not show a significant difference with BMI in the total population, according to Pearson's chi-square test. But, consumption of potato $(\mathrm{p}=0.002)$, yogurt $(0.046)$ and fried foods $(0.033)$ shows a statistically positive relationship with $\mathrm{BMI}$ in the whole study population.

Table 3. Comparing WHR with food types in Hiththatiya Middle grama niladhari area.

\begin{tabular}{|c|c|c|c|c|c|c|c|}
\hline \multirow{2}{*}{ Food } & \multicolumn{3}{|c|}{ Population with normal WHR } & \multicolumn{3}{|c|}{ Population with abnormal WHR } & \multirow{2}{*}{ P-value } \\
\hline & $\mathbf{0}$ & 1 & 2 & $\mathbf{0}$ & 1 & 2 & \\
\hline RED RAW RICE & 1 & 41 & 5 & 10 & 65 & 29 & 0.022 \\
\hline WHITE RAW RICE & 5 & 42 & 10 & 4 & 33 & 58 & 0.020 \\
\hline FISH & 2 & 11 & 68 & 2 & 15 & 54 & 0.456 \\
\hline RED MEAT & 80 & 1 & 0 & 70 & 1 & 0 & 0.925 \\
\hline WHITE MEAT & 10 & 71 & 0 & 10 & 61 & 0 & 0.752 \\
\hline POTATO & 0 & 7 & 74 & 0 & 5 & 66 & 0.715 \\
\hline LEAVES & 0 & 5 & 76 & 0 & 4 & 67 & 0.888 \\
\hline
\end{tabular}




\begin{tabular}{llllllll}
\hline \multirow{2}{*}{ Food } & \multicolumn{2}{l}{ Population with normal WHR } & \multicolumn{3}{c}{ Population with abnormal WHR } \\
\cline { 2 - 7 } & $\mathbf{0}$ & $\mathbf{1}$ & $\mathbf{2}$ & $\mathbf{0}$ & $\mathbf{1}$ & $\mathbf{2}$ & P-value \\
\hline FRUIT & 0 & 2 & 79 & 0 & 1 & 70 & 0.639 \\
YOGHURT & 16 & 64 & 1 & 14 & 56 & 1 & 0.996 \\
FRIED FOOD & 6 & 17 & 58 & 3 & 27 & 41 & 0.062 \\
VEGETABLE OIL & 79 & 1 & 1 & 71 & 0 & 0 & 0.411 \\
\hline
\end{tabular}

(Consumption frequency: $0=$ Never $1=3$ days or less than 3 days/week $2=$ more than 3 days/week)

Regarding dietary habits in Hiththatiya Middle, there is a statistically significant difference between consumption of certain food types and WHR. Consumption of red meat, white meat, fish, grain, leaves, fruits, yogurt, fried food and vegetable oil does not show a significant difference with
WHR in Hiththatiya Middle, according to Pearson's chisquare test. There is a statistically positive relationship in the consumption of red raw rice $(\mathrm{p}=0.022)$ and white raw rice $(p=0.020)$ with WHR in Hiththatiya middle area.

Table 4. Comparing WHR with food types in Hungama grama niladhari area.

\begin{tabular}{|c|c|c|c|c|c|c|c|}
\hline \multirow{2}{*}{ Food } & \multicolumn{3}{|c|}{ Population with normal WHR } & \multicolumn{3}{|c|}{ Population with abnormal WHR } & \multirow{2}{*}{ P-value } \\
\hline & 01 & 10 & 22 & 01 & 10 & 22 & \\
\hline RED RAW RICE & 2 & 18 & 60 & 1 & 13 & 58 & 0.686 \\
\hline WHITE RAW RICE & 6 & 54 & 20 & 13 & 45 & 14 & 0.132 \\
\hline RED MEAT & 78 & 2 & 0 & 67 & 5 & 0 & 0.192 \\
\hline WHITE MEAT & 7 & 71 & 2 & 13 & 57 & 2 & 0.232 \\
\hline POTATO & 0 & 31 & 33 & 0 & 52 & 36 & 0.002 \\
\hline LEAVES & 0 & 16 & 64 & 0 & 10 & 62 & 0.318 \\
\hline FRUIT & 0 & 3 & 77 & 0 & 5 & 67 & 0.379 \\
\hline YOGHURT & 46 & 32 & 2 & 51 & 21 & 0 & 0.127 \\
\hline FRIED FOOD & 5 & 56 & 19 & 5 & 52 & 15 & 0.906 \\
\hline VEGETABLE OIL & 77 & 3 & 0 & 70 & 2 & 0 & 0.737 \\
\hline
\end{tabular}

(Consumption frequency: $0=$ Never $1=3$ days or less than 3 days/week $2=$ more than 3 days/week)

Regarding dietary habits in Hungama, there is a statistically significant difference between consumption of certain food types and WHR. Consumption of red meat, white meat, fish, grain, leaves, fruits, yogurt, fried food and vegetable oil does not show a significant difference with WHR in Hungama, according to Pearson's chi-square test. There is a statistically positive relationship between the WHR and consumption of potato $(\mathrm{p}=0.002)$ in Hungama.

\section{Discussion}

This study primarily focused on Body Mass Index (BMI), Waist circumference and Waist-Hip Ratio (WHR) in a female population in two grama niladhari areas in the southern province of Sri Lanka. Furthermore the study was conducted to assess the dietary habits of each individual, for the purpose of assessing the impact of different types of food intake on BMI, and WHR.

\subsection{Association of Body Mass Index, Waist-Hip Ratio with Obesity and Abdominal Obesity}

The mean BMI value in Hungama $\left(23.78 \mathrm{kgm}^{-2}\right)$, was higher than that of Hiththatiya $\left(23.20 \mathrm{kgm}^{-2}\right)$. But, both mean BMI and WHR values in two areas are within the healthy range as mentioned in WHO guideline. Normal BMI range given in WHO reports is $18 \mathrm{kgm}^{-2}-24.9 \mathrm{kgm}^{-2}$. Abdominal obesity is defined as Waist to Hip Ratio (WHR) above 0.85 for females [1]. According to the proposed World Health
Organization cut-off values for Asians, the percentages of Sri Lankan adults in the obese and centrally obese categories were $9.2 \%$ and $26.2 \%$, respectively [3]. But, present study shows considerably high prevalence in obese $(6.6 \%)$ and abdominal obesity $(47.0 \%)$ in the total population considering BMI and WHR. According to the BMI values prevalence of both over weight and obese are higher in Hungama than those of Hiththatiya. Percentage of people, who are having a healthy value, is greater in Hiththatiya (61.2\%).

Prevalence of underweight shows to be common for both areas and it is less than $10 \%$. This is somewhat different when compared with the prevalence of underweight (33.4\%) among adolescent females as described by a study carried out in Galle [9]. When taking both over weight and obesity together, more than $35 \%$ of women in Hungama belong to that category and may be at risk of getting obesity related health problems.

\subsection{Association of Dietary Habits with BMI and WHR}

When considering the relationship between dietary habits and anthropometric parameters, WHR shows a significant positive relationship with the intake of red raw rice and white raw rice in Hiththatiya middle area. Although red raw rice does not have an adverse effect on body weight profile normally, this positive relationship with WHR could be due to the quantity of rice they consumed. It is because red raw rice is the staple food of people in these study areas, so daily consuming quantity might be higher. 
According to the present study, it shows positive relationship in consumption of red raw rice, white raw rice, potato with WHR and BMI shows a positive relationship with potato, yogurt and fried food in the total population. However, a study conducted in United State of America shows a higher consumption of potatoes and French fries was associated with a modestly increased risk of type 2 diabetes in this large cohort of women. These data support a potential benefit from limiting the consumption of these foods in reducing the risk of type II diabetes. Possible adverse effects of high potato consumption on the risk of type II diabetes because they contain large amounts of rapidly absorbed starch and thus are important contributors to dietary glycemic index [10].

Present study doesn't show any significant relation in the consumption of fruits with both BMI and WHR. As mentioned in another study carried out by the University of Lausanne, Switzerland, it is because when fruit sugar (fructose) undergoes metabolism there is a rapid hepatic conversion into glucose, glycogen, lactate, and fat. Epidemiological studies show growing evidence that consumption of sweetened beverages (containing either sucrose or a mixture of glucose and fructose) is associated with a high energy intake and increased body weight [11].

Another study carried out in Delhi, India, has investigated the changes in BMI status of women according to lifestyle and dietary habits from 329 women as a follow-up of NFHS2 Delhi sample. According to that study, there was a significant positive relationship between consumption of curd, meat, fish, fried food and fruit and a change in BMI status [12]. This result is differ from the present study which doesn't show any relationship between BMI and consumption of fish, meat, fried food or fruit when considering the whole study population. But the consumption of yogurt shows a positive relationship with BMI in the present study. However, waist-hip ratio has been found to be a stronger independent risk factor than BMI [13].

\section{Conclusion}

The results show a statistically significant positive relationship in the consumption of some food types with anthropometric parameters such as BMI and WHR in the total population. Since WC and WHR are indicators of health risk associated with excess fat around the waist, these data support a potential benefit from limiting the consumption of these foods in reducing the risk of obesity related cardiovascular diseases. People consume different types of food items, but the frequency of consumption is low for certain food Types. As total study population contains nearly $47 \%$ of abdominally obese women, proper control of body fat distribution by means of regular exercise or by having a balanced diet should be encouraged. During this study we did not conduct a dietary survey regarding the quantity of certain food types consumed by the study population. Thus we were not able to assess the relationship between the quantity of food types consumed and the parameters that we investigated in this study.

\section{References}

[1] World Health Organization (WHO), "Appropriate body-mass index for Asian populations and its implications for policy and intervention strategies", Report of a WHO Expert Consultation, The Lancet, 2004, 363.

[2] World Health Organization (WHO), "Waist-Circumference and Waist-Hip Ratio", Report of a WHO Expert Consultation, Geneva, 2011, 14.

[3] Katulanda P, Jayawardena MA, Sheriff MH, "Prevalence of overweight and obesity in Sri Lankan adults", 2010, 11(11), 751-6.

[4] Ramachandran A, Snehalatha C, "Rising burden of obesity in Asia, Journal of Obesity", E-pub 2010, 1-8.

[5] Leung SS, Ng MY, Lau TF, "Prevalence of obesity in Hong Kong children and adolescents aged 3-18 y", International Journal of Obesity, 2001, 25, 1689-1697.

[6] Al-Hilli M N and Al-Shalah H H, "Procalcitonin as a Mediator of Chronic Inflammation in Obese Women with PCOS", Medical Journal of Babylon, 2010, 7, 3-4.

[7] Ho SC, Chen YM, Woo JL, Leung SS, Lam TH, Janus ED, "Association between simple anthropometric indices and cardiovascular risk factors", International Journal of Obesity, 2001, 25, 1689-1697.

[8] Golay A, Allaz AF, Morel Y, de Tonnac N, Tankova S, Reaven G, "Similar weight loss with low- or high-carbohydrate diets", American Journal of Clinical Nutrition, 1996, 63, 174-8.

[9] Hettiarachchi M, Liyanage C, Wickremasinghe R, David C, Steven A. "Prevalence and severity of micronutrient deficiency: a cross-sectional study among adolescents in Sri Lanka" Asia Pacific Journal of Clinical Nutrition, 2006, 15 (1), 56-53.

[10] Thomas L, Walter C, Simin L, JoAnn E, "Possible adverse effects of high potato consumption on the risk of type II diabetes because they contain large amounts of rapidly absorbed starch and thus are important contributors to dietary glycemic index", American Journal of Clinical Nutrition, 2006 , vol. 83, 284-290.

[11] Tappy L, Le K. A, "Metabolic effects of fructose and the worldwide increase of obesity", Physiological Reviews, 2010, $90(1), 23-46$

[12] Agrawal $\mathrm{P}$ et al, "Role of lifestyle and diet in emerging obesity among Indian women and its impact upon their health status", International Union for the Scientific Study of Population Paper, $25^{\text {th }}$ International Population Conference, France, 2005.

[13] Lapidus L, Bengtsson C, Larsson B, Pennert K, Rybo E, Sjostrom L, "Distribution of adipose tissue and risk of cardiovascular disease and death: a 12 year follow up of participants in the population study of women in Gothenburg, Sweden” British Medical Journal, 1984, 289 (6454), 12571261.

[14] Agrawal P and Vinod M, "Covariates of Overweight and Obesity among women in North India", East-West center working papers, Population and health series, 2004, 116. 
[15] Fernando S D, Paranawitane S R, Rjakaruna J, Weerasinghe S De Silva D, Wickramasinghe A R, "The Health and Nutritional Status of school children in two rural communities in Sri Lanka", Tropical Medicine and International Health, 2000, 5(6), 450-452.

[16] Jayathissa R, National Food Security Survey (NFSS), "Current Nutritional status of children in Sri Lanka", Sri Lanka Nutrition Bulletin, 2009, (1), 5.
[17] Misra, Shrivastava U, "Obesity and dyslipidemia in South Asians." Nutrients journal, 2013, 5(7), 2708-2733.

[18] Seidell J C, Waist circumference and waist/hip ratio in relation to all - cause mortality, cancer and sleep apnea, European Journal of Clinical Nutrition, 2010, 64(1), 35-41.

[19] Flegal, Katherine M, Carroll, Prevalence and trends in obesity among US adults, JAMA journal, 2010, 303 (3), 235-241. 